Musées, Patrimoine et Culture scientifiques et techniques

$194 \mid 2021$

mars-avril 2021

\title{
Le projet européen SensMat. Accompagner la conservation préventive dans les musées
}

\section{Marie-Dominique Bruni}

\section{OpenEdition \\ Journals}

Édition électronique

URL : https://journals.openedition.org/ocim/4269

DOI : 10.4000/ocim.4269

ISSN : 2108-646X

Éditeur

OCIM

Édition imprimée

Date de publication : 1 mars 2021

Pagination : 38-43

ISSN : 0994-1908

Référence électronique

Marie-Dominique Bruni, « Le projet européen SensMat. Accompagner la conservation préventive dans les musées ", La Lettre de I'OCIM [En ligne], 194 | 2021, mis en ligne le 01 mars 2021, consulté le 11 avril 2022. URL : http://journals.openedition.org/ocim/4269 ; DOI : https://doi.org/10.4000/ocim.4269

Ce document a été généré automatiquement le 11 avril 2022.

Tous droits réservés 


\section{Le projet européen SensMat. Accompagner la conservation préventive dans les musées}

Marie-Dominique Bruni

Contrôler à distance les conditions de conservation des collections.

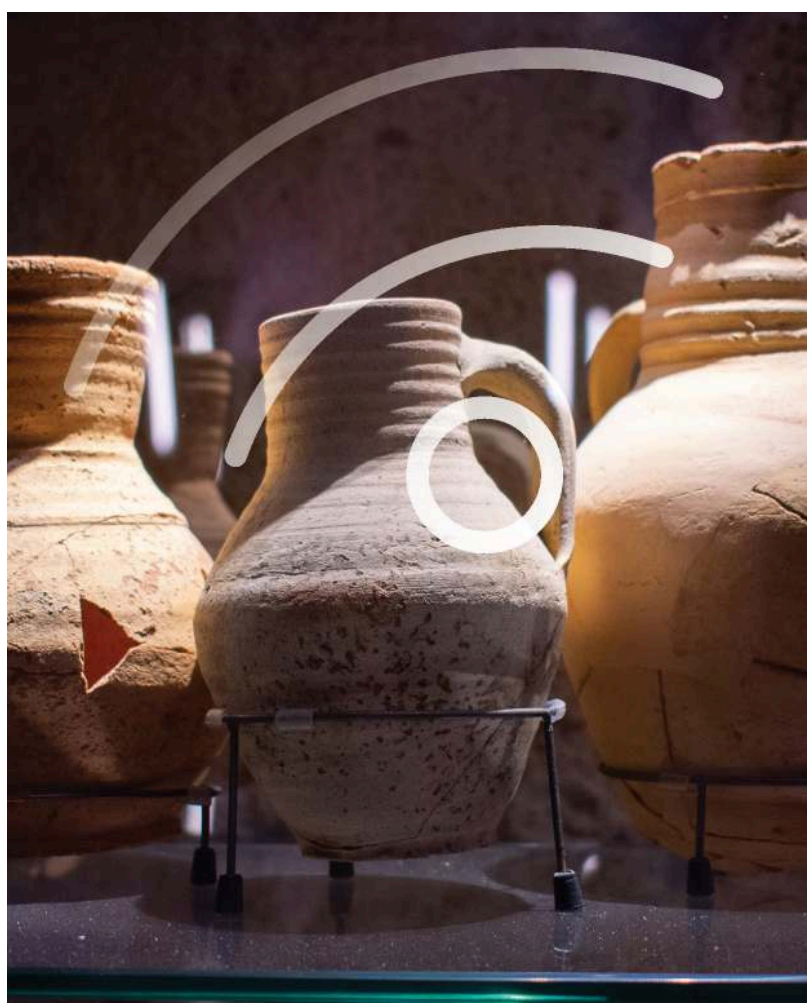

(c) Ocim/inmorino/Pixabay 
1 La richesse culturelle et touristique du patrimoine culturel européen est unanimement reconnue ${ }^{1}$. Néanmoins, de plus en plus d'institutions (Icom, Unesco, Iccrom, etc.) alertent sur la nécessité urgente de sauver de nombreuses collections fragiles d'un environnement inadapté.

2 Depuis quelques décennies, les professionnels du patrimoine développent des actions et des politiques de conservation préventive. Les connaissances dans ce domaine s'étoffent, notamment par rapport aux spécificités de la grande variété des matériaux et des lieux de conservation. Et les moyens d'observation et de contrôle des conditions climatiques des collections se multiplient. Mais les professionnels se heurtent bien souvent à la difficulté d'interpréter ces données pour adapter leurs choix et faire évoluer leurs pratiques.

\section{L'approche SensMat}

3 L'enjeu principal de SensMat est de proposer un système de surveillance totalement intégré et de fournir des outils de gestion des données et des outils d'aide à la décision.

Il s'agit d'une solution de conservation préventive conviviale, de type "tout-en-un ». Dans le projet, les acteurs technologiques et culturels relèvent ensemble les défis de conservation préventive, afin d'aider les petits et moyens musées à s'équiper. La contribution de l'Europe, pour ce projet, se monte à environ 6 millions d'euros. Le projet a été lancé en janvier 2019 pour une durée de trente-huit mois, c'est-à-dire jusqu'au 28 février $2022^{2}$.

5 La solution SensMat intégrera les derniers capteurs avancés pour fabriquer des systèmes de détection bon marché et intégrés en vue de la production de masse. Le système capitalisera sur les dernières avancées des systèmes intelligents connectés (Internet des objets - IoT) et de la gestion de bases de données complexes via l'intelligence artificielle (Multi-Criteria-Decision-Making-MCDM). 


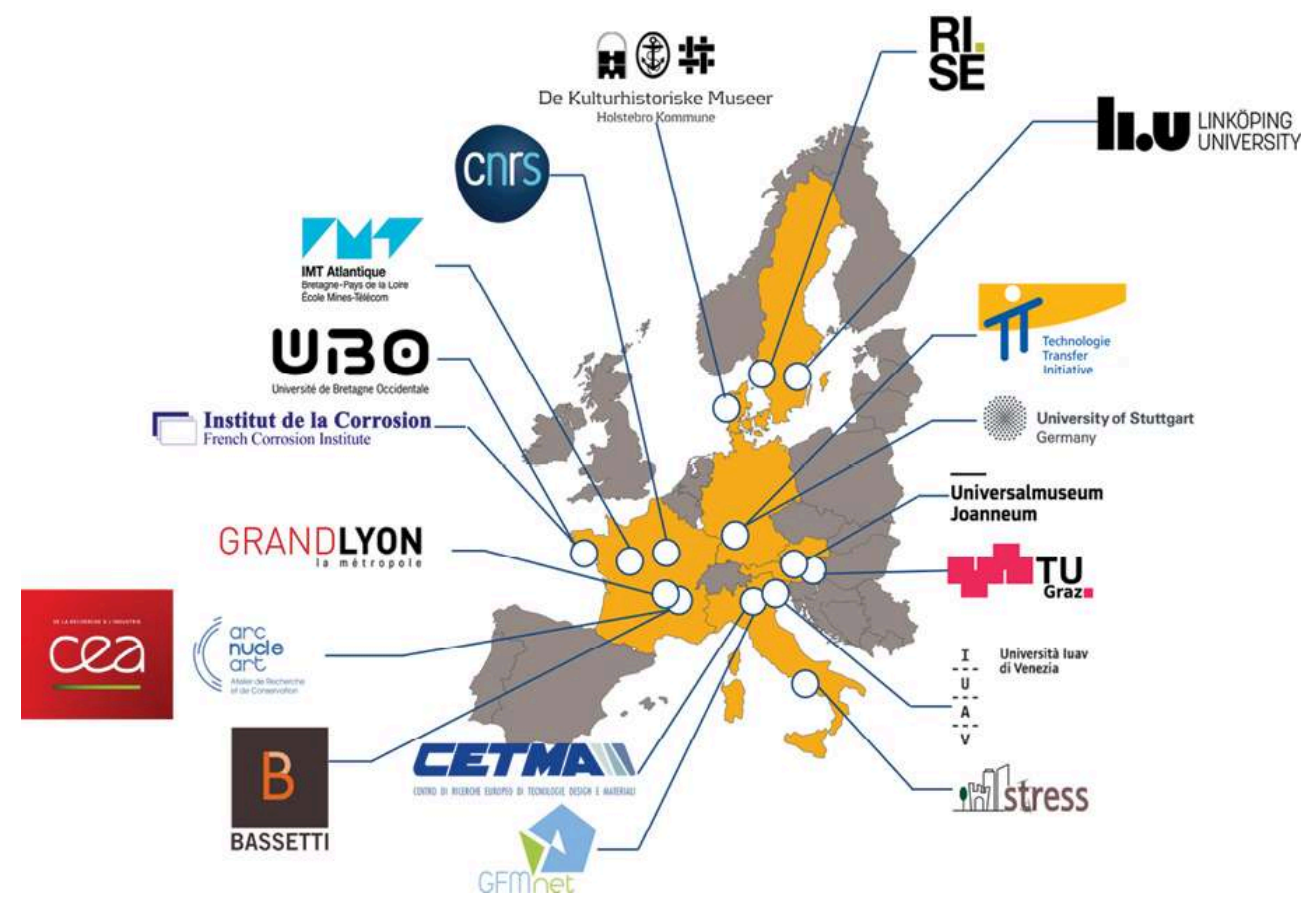

(C) DR

6 Afin d'atteindre les objectifs ci-dessus, les dix-huit partenaires, répartis dans six pays européens, investiguent trois domaines : les capteurs, la modélisation et la gestion des connaissances et des données.

Ils doivent relever six objectifs techniques principaux :

8 - permettre à tous les musées de déployer des solutions environnementales évolutives, connectées et rentables de surveillance ;

9 - développer et lier des modèles multi-physiques et multi-échelles d'environnement et de dégradation ;

10 - développer des capteurs sans contact pour la surveillance des matériaux ;

11 - préparer des outils d'aide à la décision ;

12 - concevoir et communiquer des recommandations, des stratégies de gestion de la conservation et des évolutions de normalisation;

13 - démontrer les performances et viser une maturité technologique de TRL 7 (Technology Readiness Level). Pour atteindre cette maturité il faut intégrer la solution prototype dans un environnement opérationnel (proche de l'environnement réel final et associé aux systèmes auxiliaires éventuels). Le prototype est à une échelle proche du système opérationnel, la plupart des fonctions sont disponibles. Il s'agit par ailleurs de fournir le service associé pour la démonstration de l'innovation c'est-à-dire de livrer une première documentation du prototype et de former les utilisateurs.

\section{De la captation des données aux recommandations}

Le projet vise à apporter une solution centrée et adaptée au besoin du futur utilisateur. Il doit proposer des outils innovants moins chers que les solutions existantes, avec des 
interfaces conviviales, utilisant des capteurs discrets d'un point de vue visuel, pouvant aider à l'évaluation des risques et à la prévention des dommages liés à des facteurs environnementaux. SensMat aidera à contrôler et surtout alerter sur les conditions de conservation des collections. Pour soutenir les conservateurs dans leurs actions de prévention et les assister dans leur prise de décision, les outils informatiques d'aide à la décision seront intégrés et adaptés au cas par cas afin de compléter la solution globale finale.

Ainsi la solution combinera les derniers développements, sur les capteurs de mesure de température $\left(\mathrm{T}^{\circ} \mathrm{C}\right)$, humidité relative ( $\left.\mathrm{RH} \%\right)$, composés organiques volatils (COV), corrosivité, poussières (particules), lumière, vibration. Elle conjuguera aussi les récentes avancées : en modélisation de la dégradation des matériaux, modélisation d'un bâtiment musée ou d'une salle de musée (l'enveloppe et le climat interne, avec le BIM Building Information Modeling), les progrès de l'IoT (Internet of Things, internet des objets). L'IoT est un écosystème qui permet l'interconnexion physique et virtuelle, de « devices - objets» qui sont connectés entre eux et aussi à Internet. Il est ainsi possible de connecter des lieux, des capteurs (enregistrant des données), des réseaux (transmettant les données), des outils de management de la connaissance et d'aide à la décision (DMT). Les objets connectés à l'Internet permettent alors une communication entre nos biens physiques et leur existence numérique pour rassembler, stocker, gérer de nouvelles données, connaissances et savoirs sur le réseau.

Modélisation, de la captation des données aux recommandations.

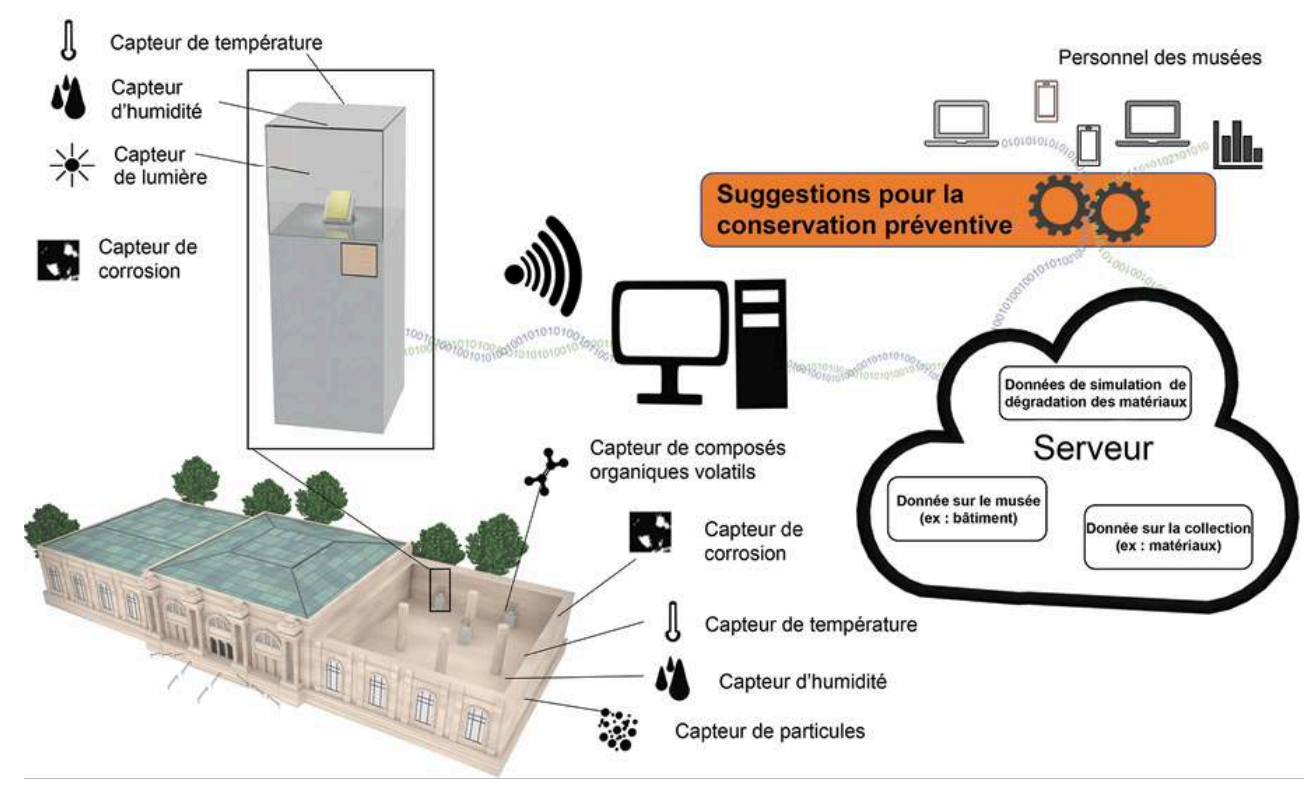

(c) DR

L'objectif est d'adapter ces innovations aux besoins de chaque musée, à ses contraintes et ses acteurs (selon la sensibilité des matériaux, pour les objets et collections dont les musées proposent le suivi, en fonction des contraintes juridiques, sociétales, environnementales, culturelles...). Les outils BIM par exemple apporteront une connaissance en vue de mieux maîtriser les conditions environnementales : l'interface BIM est une interface interactive qui facilitera la mise en œuvre des technologies (capteurs...) et des méthodes nécessaires à la conservation préventive. 
17 Ainsi, les utilisateurs pourront naviguer et interagir avec des modèles tridimensionnels (3D) dont les informations seront couplées aux informations sur le bâtiment (enveloppe du bâtiment, fenêtres, ventilation...), aux informations sur les objets à surveiller et aux informations enregistrées en temps réel par les capteurs.

Grâce à la capitalisation des connaissances avec la base de données techniques et d'informations «contextuelles", la solution SensMat facilitera la diffusion (communication et transfert de connaissance et compétences) et contribuera à la maitrise (outil d'aide à la décision) des actions de prévention. Par exemple l'exploitation des données permettra d'établir des «liens de causes à effets des dégradations » de certains matériaux sur des objets identifiés comme sensibles par les acteurs de la conservation des musées, de les répertorier, analyser, tout ceci en tenant compte des recommandations et normes internationales, des politiques de conservation nationales ou régionales. L'automatisation de la collecte de données via ces systèmes de plateformes et la proposition des outils de management de la connaissance seront proposés avec le service adapté ce qui constituera l'offre complète SensMat.

Des formations et recommandations sur les meilleures pratiques sont prévues et complèteront les démonstrations pour prouver la faisabilité et les performances de la solution. L'objectif est de traiter l'ensemble de la chaîne d'information de la conservation jusqu'à la normalisation, en aidant le personnel des musées à relever des défis comme le choix des meilleures conditions d'exposition, l'élaboration de la meilleure stratégie de mise en place des outils de surveillance, des actions de maintenance sécurisées et de faciliter des actions de «standardisation-normalisation » de nouvelles méthodes de conservation.

$\mathrm{Au}$ cours du projet, toute la chaîne de valeur de la solution SensMat sera prise en compte, analysée et approfondie avec l'ambition de définir la valeur d'usage directe mais aussi la valeur indirecte. En effet, avec les partenaires technologiques et culturels, SensMat vise à définir une approche parallèle et complémentaire pour évaluer l'impact généré par les valeurs indirectes. Cette activité sensibilisera les partenaires à d'autres avantages potentiels, des besoins secondaires, provenant des commentaires des utilisateurs des solutions et services alors développés. L'idée principale est de ne pas considérer l'utilisateur final dans un rôle passif, mais de l'impliquer dans la création de valeur ajoutée en exploitant conjointement les opportunités. Ce «clustering " vise à soutenir une nouvelle approche sur l'évaluation des technologies au sein des paradigmes actuels et futurs, afin de combiner des résultats différents et intersectoriels partageant la même portée pour faciliter et maximiser l'impact global, une valeur ajoutée supplémentaire, une meilleure exploitation et compétitivité sectorielle.

\section{Le Musée dauphinois, site test du projet SensMat}

Pratiquer la conservation préventive dans un bâtiment complexe, un ancien couvent du XVII ${ }^{e}$ siècle classé monument historique non climatisé, c'est le défi relevé chaque jour par l'équipe du Musée dauphinois! Avec deux à trois expositions temporaires par an, des expositions de longue durée et des réserves conséquentes, la préservation des collections est au cœur des missions du musée, ce qui demande du suivi et de la réactivité. 
Partenaire historique du Département de l'Isère, Arc-Nucléart a notamment contribué à l'extraordinaire chantier de sauvetage et de préservation des objets néolithiques et médiévaux sortis du lac de Paladru, conservés et valorisés aujourd'hui par le Musée dauphinois. Fort de ce lien, le musée n'a pas hésité longtemps à accepter la proposition d'Arc-Nucléart de devenir un site test pour le projet SensMat. Prendre part à ce projet de recherche européen ambitieux et concret est un honneur, une chance aussi. C'est l'opportunité pour le Musée dauphinois d'acquérir une fine connaissance de son environnement dans toutes ses composantes (climat, poussière, vibrations, émanations, lumière...) et de bénéficier de conseils opérationnels et pratiques sur mesure pour une gestion et une préservation optimales des collections.

Fabienne Pluchart, Responsable du pôle collections/documentation au Musée dauphinois, Grenoble

\section{Le programme SensMat à mi-parcours}

21 Les résultats à mi-parcours ont été soumis à la commission Européenne en octobre 2020 et ont reçu une évaluation encourageante.

Dans un premier temps, une enquête à grande échelle sur l'évolution des besoins, des barrières, des normes, de la législation, etc. a été réalisée et un panel de musées européens a pu répondre à un questionnaire dédié ${ }^{3}$. En tenant compte des recommandations et normes internationales, des politiques de conservation nationales ou régionales, le consortium SensMat a défini les spécifications techniques, économiques, ergonomiques et esthétiques pour concevoir tous les outils de conservation préventive nécessaires à la mise en œuvre d'une politique de conservation préventive efficace, dans les petits et moyens musées, et facilement accessibles aux utilisateurs non scientifiques. 


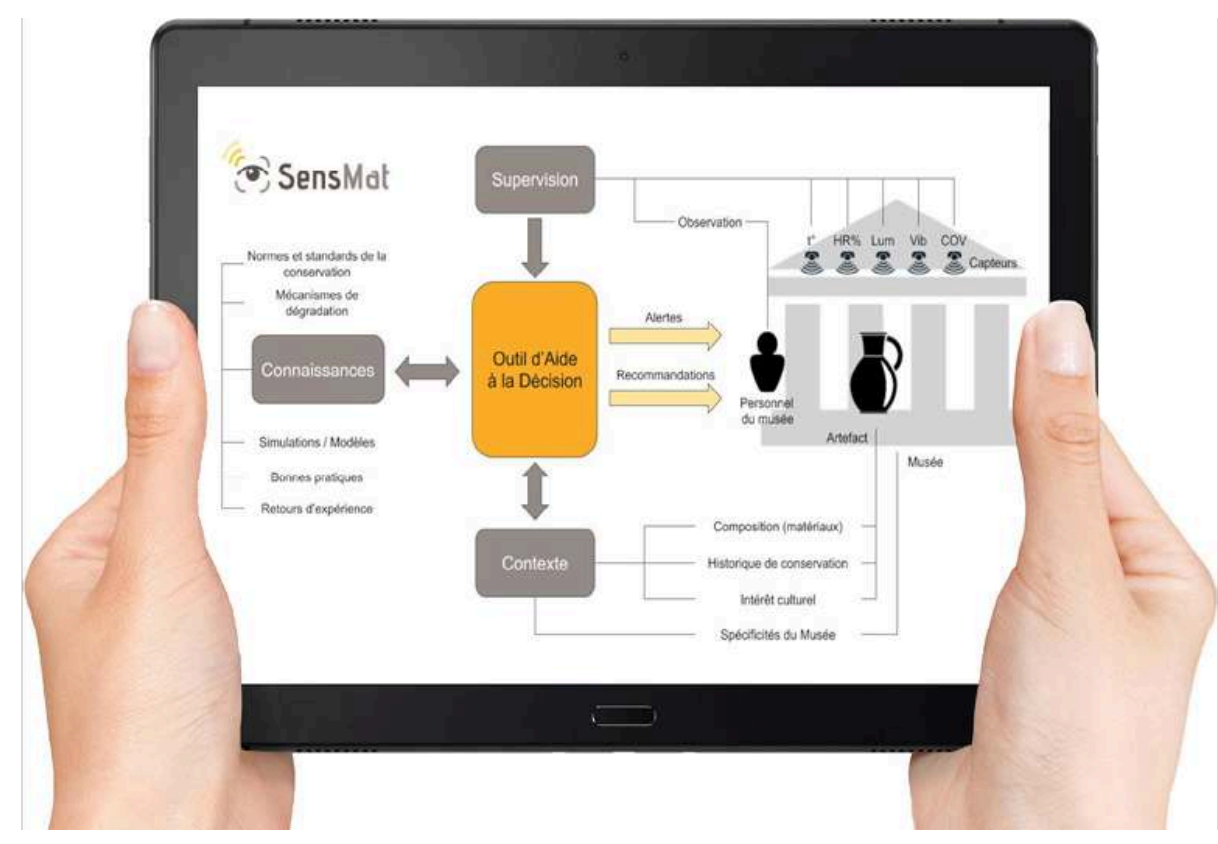

(c) DR

La modélisation des phénomènes de dégradation par rapport aux conditions environnementales, sera un des principaux outils de prédiction à partir des données des capteurs. Des travaux de caractérisation ont été menés pour valider les modèles (analyse de matériaux, spectroscopie Raman portable, caméra de thermographie 2D Infra Rouge).

4 Les résultats de la modélisation, les données et les métadonnées associées, seront stockés dans des référentiels de données. Les ensembles de données de la solution SensMat sont décrits de manière à être faciles à trouver et à partager. Une interface de programmation applicative (API) offre la possibilité d'automatiser le téléchargement et l'accès aux données.

Le développement et la caractérisation des capteurs ont été réalisés selon deux axes de développement:

- plateformes intégrées de capteurs sans fil intelligents basés, lorsque cela est possible, sur une technologie imprimée hybride ou, dans des circuits imprimés alternatifs, à faible coût ;

• «capteurs de dégradation" individuels sans contact spécifiques pour détecter en temps réel la dégradation de l'artefact lui-même. Les techniques impliquent la thermographie IR, la mesure par radiofréquence sur des feuilles métalliques.

28 Trois différents types de systèmes prototypes de capteurs ont été développés :

- 2 plateformes multi-capteurs sans fil différentes :

- plateforme de détection imprimée hybride pour $\mathrm{HR} \%, \mathrm{~T}^{\circ} \mathrm{C}$ et corrosivité ;

- plateforme de capteurs sans fil à faible coût.

- 3 systèmes de capteurs améliorés différents :

- système de capteur COV ;

- système de capteurs de particules PM (Particulate Matters / matières particulaires) ; 


\section{Le projet SensMat se poursuit}

37 La préparation de l'utilisation des résultats dans les études de cas a commencé, et les activités pour les démonstrations sont lancées. Il y a eu quelques évolutions pour les 10 cas d'études ou démonstrateurs sélectionnés par rapport aux cas d'études initiaux choisis dans la proposition initiale et des discussions importantes ont eu lieu pour sélectionner les typologies de collections. En effet, il fallait garantir dans la mesure du possible un large éventail pour prendre en compte des cas représentatifs en Europe (climats différents).

nous permettre d'optimiser les outils, la qualité, la fiabilité et la convivialité. Les activités de communication, comme l'organisation d'ateliers avec des acteurs clefs européens, l'analyse approfondie des retours des musées partenaires, des utilisateurs des dix premiers démonstrateurs, du conseil d'experts européens SensMat, permettront de faire évoluer la maturité de la solution SensMat.

La processus d'exploitation est en cours de démarrage et la feuille de route précise est en cours de définition. Nous avons initié une étude de marché afin d'affiner le plan d'exploitation futur et la feuille de route détaillée.

L'auteure remercie Fabienne Pluchart du Musée dauphinois, le CEA/Arc-Nucléart ainsi que Absiskey, consultant qui accompagne la coordination du projet.

\section{NOTES}

1. L'Organisation de coopération et de développement économiques (OCDE) affirme que ce patrimoine culturel permet d'assurer $50 \%$ de l'activité touristique et génère directement et indirectement des millions d'emplois locaux. Voir Tendances et politiques du tourisme de l'OCDE 2020 : https://www.oecd.org/industry/tourism/TENDANCES-ET-POLITIQUES-DU-TOURISME-DELOCDE-2020.pdf

2. Le projet à l'origine de cette candidature a reçu un financement du programme de recherche et d'innovation Horizon 2020 de l'Union européenne au titre de la subvention n ${ }^{\circ} 814596$.

3. Voir SensMat Deliverable D2.4 Questionnaire :

https://www.sensmat.eu/wp-content/uploads/2019/10/SensMat_D2.4_M6_vfinal.pdf

La Lettre de I'OCIM, 194 | 2021 


\section{RÉSUMÉS}

Durant trois ans, dix-huit acteurs culturels et scientifiques collaborent au projet européen SensMat, lié à la conservation préventive. L'objectif est de concevoir un outil d'accompagnement et d'aide à la décision pour les personnels des musées. À mi-parcours, le coordinateur du projet présente les objectifs, les ambitions et les étapes à venir.

INDEX

Mots-clés : conservation préventive, métier/personnel des musées

\section{AUTEUR}

\section{MARIE-DOMINIQUE BRUNI}

Program manager au CEA Commissariat à l'énergie atomique et aux énergies alternatives à Grenoble, coordinateur du projet SensMat.

marie-dominique.bruni@cea.fr 